\title{
Preferences Stability: A measure of preferences changes over time
}

\author{
R. de Andrés Calle ${ }^{\mathrm{a}, *}$, J.M. Cascón ${ }^{\mathrm{b}}$, T. González-Arteaga ${ }^{\mathrm{c}}$ \\ ${ }^{a}$ BORDA Research Unit, PRESAD Research Group and \\ Multidisciplinary Institute of Enterprise (IME), University of Salamanca, \\ E37007 Salamanca, Spain \\ ${ }^{b}$ Department of Economics and Economic History, \\ Institute on Fundamental Physics and Mathematics University of Salamanca, \\ E37007 Salamanca, Spain \\ ${ }^{c}$ BORDA and PRESAD Research Groups, University of Valladolid, \\ E47011 Valladolid, Spain
}

\begin{abstract}
Traditionally, preferences have been considered stable although there are growing evidences that such stability is a mere theoretical assumption. Attending to this fact, it should be interesting to measure how much stability preferences provide in order to improve decision making processes. Surprisingly, no research has been found on measuring preferences stability.

To overcome this drawback, this paper proposes a novel approach for measuring the stability of preferences and also for improving understanding of current and future decisions. In order to be faithful to reality, this research considers decisions like complete pre-orders on a set of alternatives. Following this reasoning, this paper provides the general concept of decision stability measure as well as two specific measures: the local and the global decision stability measure. More-

\footnotetext{
${ }^{*}$ Corresponding author

Email addresses: rocioac@usal.es (R. de Andrés Calle), casbar@usal.es (J.M. Cascón), teresa.gonzalez . arteaga@uva.es (T. González-Arteaga)
} 
over, the main features of the novel approach are examined, including several mathematical results on the behaviour of the proposed measure. And eventually, this contribution develops two real cases of study, with in-depth analysis of preferences behaviour and their stability over time. Specifically, the first one explores into the characteristics of Spanish citizens' voting behaviour and the second one attempts to analyse European citizens' preferences about passenger car market. Keywords: Preference stability, decision stability, complete pre-orders, loss memory effect.

\section{Introduction}

Every day people deal with decisions like paying by credit card or by cash, going on holidays or increasing their pension fund, working or studying, and so on. These routine decisions and their stability over time have become a central issue in the actual global world due to the fact that the assumption of stability of preferences is a key aspect to make accurate market studies and predictions. To extract conclusions from previous decisions to come decisions, it is necessary to suppose that individuals make such decisions on basis to the same rules over time.

Imagine, for example, that the manager team of a restaurant wants to change the daily menu and they ask for customers' preferences on the actual daily menu during one year. To ensure a right change in the daily menu, team manager needs not only to know what the customers' preferences are but need to know if these preferences will be stable over time. At first sight one may think that if the customers' preferences are not the same every time i.e., select the same dishes, their preferences are not stable, but are not necessarily so.

Suppose three customers that rank the starters of the daily menu (seafood 
chowder, roast vegetarian, caesar salad and stag's grilled) in the following way for three periods of time:

\begin{tabular}{|c|c|c|c|}
\hline & Customer 1 & Customer 2 & Customer 3 \\
\hline Period 1 & $\begin{array}{l}\text { roast vegetarian } \\
\text { seafood chowder } \\
\text { caesar salad, stag's grilled }\end{array}$ & $\begin{array}{l}\text { stag's grilled } \\
\text { caesar salad } \\
\text { seafood chowder } \\
\text { roast vegetarian }\end{array}$ & $\begin{array}{l}\text { caesar salad } \\
\text { seafood chowder } \\
\text { stag's grilled } \\
\text { roast vegetarian }\end{array}$ \\
\hline Period 2 & $\begin{array}{l}\text { roast vegetarian } \\
\text { seafood chowder } \\
\text { caesar salad, stag's grilled }\end{array}$ & $\begin{array}{l}\text { caesar salad } \\
\text { seafood chowder } \\
\text { roast vegetarian } \\
\text { stag's grilled }\end{array}$ & $\begin{array}{l}\text { roast vegetarian } \\
\text { caesar salad } \\
\text { seafood chowder } \\
\text { stag's grilled }\end{array}$ \\
\hline Period 3 & $\begin{array}{l}\text { seafood chowder } \\
\text { roast vegetarian } \\
\text { caesar salad, stag's grilled }\end{array}$ & $\begin{array}{l}\text { caesar salad } \\
\text { seafood chowder } \\
\text { roast vegetarian } \\
\text { stag's grilled }\end{array}$ & $\begin{array}{l}\text { stag's grilled } \\
\text { roast vegetarian } \\
\text { caesar salad } \\
\text { seafood chowder }\end{array}$ \\
\hline
\end{tabular}

Table 1: Customers' preferences for three periods of time.

To take a decision, the manager team needs to establish which of the customers' preferences are more stable although this fact is not obvious. As can be seen from Table 1, Customer 1 keeps her/his preferences in the periods 1 and 2 , but changes them in the period 3. Customer 2 keeps her/his preferences stable in the period 2 and 3, but changes in the period 1. And finally, Customer 3 changes her/his preferences in every period of time. How much stability do these customers' preferences contain? Is it possible to measure this stability? Does customers' preferences stability depend on the period of time in which there are preferences changes? The challenge of this paper is to answer these opened questions by means of defining a mathematical instrument able to measure the stability 
of the customers' preferences and consequently, to make manager team's decision easier. Therefore, the central thesis of this paper is proposed a novel methodology for measuring preference stability over time and also for improving understanding of preferences behaviour when preferences are considered like complete pre-orders (to represent faithfully individuals' preferences).

This study therefore sets out a general definition of decision stability measure that includes two minimum conditions, full decision stability ${ }^{1}$ and decision stability neutrality ${ }^{2}$ in order to develop a deep and strong general mathematical measure. In addition, this study contributes to this growing area of research by providing two particular decision stability measures: the local decision stability measure and the global decision stability measure. The study of such measures is completed including the main features of the novel approaches as well as several mathematical results on the proposed measures behaviour. Finally, it is hoped that this research will contribute to a deeper understanding of the proposed measures including two real cases of study. Specifically, the first one explores into the characteristics of Spanish citizens' voting behaviour and the second one attempts to analyse European citizens' preferences about passenger car market.

This work is composed of seven sections. The first section of this paper, Section 2, examines different related approaches in literature. Section 3 gives a brief overview of the notation as well as some basic definitions. Section 4 is concerned with the novel general measure proposed in this study as well as two specific ones: the local decision stability measure and the global decision stability measure. Section 5 analyses the main features of the novel approaches as well as

\footnotetext{
${ }^{1}$ The measure of stability must be maximum if preferences over time are equal.

${ }^{2}$ The measure of stability must not change if the alternatives are permuted.
} 
several mathematical results on the proposed measures behaviour ${ }^{3}$. Section 6 develops two real cases of study, with in-depth analysis of preferences behaviour and of measuring their stability. Finally, Section 7 presents the findings and the conclusions of the research.

\section{Related research}

The stability of preferences has long been a question of great interest in a wide range of fields like Economic Analysis, Psychology and Decision Making Analysis and its interest has grown extensively over the last 25 years.

Traditionally and under the perspective of Economic Analysis (Neoclassical Economic Theory), changes in consumers' choices over time cause changes in relative prices but not on consumers' preferences that remain stable over time. In the seminal contribution of Stigler and Becker (1977) [1], consumers' preferences should be considered stable and if there are differences among the observed choices over time, these do not lead to an inconsistency. They defended that preferences must be defined over different state spaces and consequently, the choices are associated to such spaces.

More recently, experimental literature has emerged that offers conflicting findings about this conventional assumption (an extensive review of such literature can be found on [2]). In particular, there are evidences of stability in risk preferences ([3], [4], [5] and [6]), social preferences ([7], [8], [9] and [10]) and time preferences ([11], [12] and [10])).

\footnotetext{
${ }^{3}$ To keep the paper to a manageable size and to maintain an overall view of the results, all proofs have been omitted but they are available under request.
} 
From Psychology perspective, it is assumed that some human behaviours play an enduring part of human thoughts and feelings. In this type of literature, decision makers' choices and behaviours are based on measuring the aforementioned personality characteristics. In the same way that Economics Analysis research, these studies on the temporal stability of personality traits do not reach any decisive conclusion (see [13], [14], [15] and [16], among others).

The growing studies under Decision Making perspective examine preferences stability by means of analysing the consistency of the decision makers choices ([17], [18], [19], [20], [21], [22] and [23]). To conclude, Table 2 summarises and classifies the approaches that have been reviewed.

Based on the aforementioned literature, it could be appealing and necessary to study and to measure how much stability preferences provide. To our knowledge, few previous studies have investigated the measurement of preferences stability (see [24], [25] and [26]). A seminal study in this issue is the work of GonzálezArteaga, de Andrés Calle and Peral [24]. In this contribution authors proposed a new measure of preference stability under the assumption of dichotomous opinions i.e., individuals approve or disapprove alternatives at different moments of time. Specifically, authors considered the notion of preference stability similar to the notion of preferences cohesiveness. This methodology was applied to measure the stability of patients' preferences for life-sustaining treatments.

In subsequent papers ([25] and [26]), authors introduced a similar measurement to the one in [24] but in these cases under the assumptions of fuzzy preferences and preferences defined in the unit interval, respectively. By means of assuming fuzzy preferences authors improved the previous work due to evidence suggests that humans may experience difficulties in expressing uncertain know- 
Preferences Stability under Economics perspective

\begin{tabular}{|c|c|}
\hline Author(s) & Hypothesis \\
\hline Stigler and Becker [1] & Stability of preferences \\
\hline \multicolumn{2}{|l|}{ Andersen et. al. [3] } \\
\hline \multicolumn{2}{|l|}{ Harrison, Lau and Yoo [4] } \\
\hline \multicolumn{2}{|l|}{ Lönnqvis et. al.[5] } \\
\hline \multicolumn{2}{|l|}{ Zeisberger, Vrecko and Langer [6] } \\
\hline \multicolumn{2}{|c|}{ Carlsson, Johansson-Stenman and Nam [7] } \\
\hline \multicolumn{2}{|l|}{ de Oliveira, Eckel and Croson [8] } \\
\hline \multicolumn{2}{|l|}{ Levitt and List [9] } \\
\hline \multicolumn{2}{|l|}{ Volk, Thöni and Ruigrok [10] } \\
\hline \multicolumn{2}{|c|}{ Carlsson, Johansson-Stenman and Nam [11] } \\
\hline Tanaka, Camerer and Nguyen [12] & Stability of temporal preferences \\
\hline \multicolumn{2}{|l|}{ Volk, Thöni and Ruigrok [10] } \\
\hline \multicolumn{2}{|c|}{ Preferences Stability under Psychology perspective } \\
\hline Author(s) & Hypotheses \\
\hline \multicolumn{2}{|l|}{ Cobb-Clark and Schurer [13] } \\
\hline \multicolumn{2}{|l|}{ Cobb-Clark and Schurer [14] } \\
\hline \multicolumn{2}{|l|}{ Borghans et. al. [15] } \\
\hline \multicolumn{2}{|l|}{ Heckman, Pinto, and Savelyev [16] } \\
\hline \multicolumn{2}{|c|}{ Preferences Stability under Decision Making perspective } \\
\hline Author(s) & Hypothesis \\
\hline \multicolumn{2}{|l|}{ Arnott and Gao [17] } \\
\hline \multicolumn{2}{|l|}{ Brandts and Charness [18] } \\
\hline \multicolumn{2}{|l|}{ Crosetto and Filippin [19] } \\
\hline Dave et. al. [20] & Stability of decision makers' choices \\
\hline \multicolumn{2}{|l|}{ Ding et. al [21] } \\
\hline \multicolumn{2}{|l|}{ Geiger and Schader [22] } \\
\hline Lim and Lee [23] & \\
\hline
\end{tabular}

Table 2: Summary table of studies related to preferences stability 
ledge in a dichotomous way [27].

\section{Notation and starting hypothesis}

Let $\mathbf{N}=\{1, \ldots, n\}$ a society of individuals and $\mathbf{X}=\left\{x_{1}, \ldots, x_{k}\right\}$ a finite set of alternatives, $|\mathbf{X}| \geq 2$. There is no loss of generality in assuming that the individuals and/or the society ranks the alternatives by means of complete preorders $^{4}$ on $\mathbf{X}$. This assumption allows to reflect the reality of decision lifesituations. The set of all complete preorders on $\mathbf{X}$ will be denoted by $\mathbf{W}(\mathbf{X})$.

Let $\mathbf{T}=\left\{t_{0}, \ldots, t_{T}\right\}$ be an ordered time sequence, namely, a temporal set ${ }^{5}$. Let $\mathcal{R}_{i} \in \mathbf{W}(\mathbf{X})$ be a temporal decision ${ }^{6}$ on the set of alternatives $\mathbf{X}$ at the moment of time $t_{i} \in \mathbf{T}$. The notation $x_{k} \mathcal{R}_{i} x_{j}$ means that the alternative $x_{k}$ is at last as good as $x_{j}$ at the moment of time $t_{i}$.

Let $\mathcal{P}=\left(\mathcal{R}_{0}, \mathcal{R}_{1}, \ldots, \mathcal{R}_{T}\right) \in \mathbf{W}(\mathbf{X}) \times \ldots \times \mathbf{W}(\mathbf{X})=\mathbf{W}(\mathbf{X})^{T+1}$ be a temporal decision profile on the set of alternatives $\mathbf{X}$ over $T+1$ moments of time. The $\mathcal{R}_{i} \in \mathcal{P}$ profile element is a temporal decision on $\mathbf{X}$ at the moment of time $t_{i}, i \in\{0, \ldots, T\}$. Let $\mathbf{P}(\mathbf{X})$ denote the set of all temporal decision profiles, that is, $\mathbf{P}(\mathbf{X})=\bigcup_{T \geq 1} \mathbf{W}(\mathbf{X})^{T+1}$.

Let $\mathcal{P}^{-1}$ denote the reversal of the temporal decision profile $\mathcal{P}$ defined by $\mathcal{P}^{-1}=\left(\mathcal{R}_{0}^{-1}, \ldots, \mathcal{R}_{T}^{-1}\right)$ where $x_{k} \mathcal{R}_{i}^{-1} x_{j} \Longleftrightarrow x_{j} \mathcal{R}_{i} x_{k}$. The temporal decision profile $\mathcal{P}$ is constant over time to $\mathcal{R}_{i}$ if $\mathcal{P}=\left(\mathcal{R}_{i}, \ldots, \mathcal{R}_{i}\right)$.

Any permutation $\pi$ of the alternatives $\left\{x_{1}, \ldots, x_{k}\right\}$ determines a permutation

\footnotetext{
${ }^{4}$ Reflexive and transitive binary relation, denoted by $\succcurlyeq$.

${ }^{5}$ It is worth pointing out that moments of time in the temporal set $\mathbf{T}=\left\{t_{0}, \ldots, t_{T}\right\}$ do not need to be equidistantly distributed.

${ }^{6}$ Note that temporal decisions can be taken by individuals or by society.
} 
of every temporal decision $\mathcal{R}_{i} \in \mathbf{W}(\mathbf{X})$ via $x_{s}{ }^{\pi} \mathcal{R}_{i} x_{t} \Longleftrightarrow x_{\pi(s)} \mathcal{R}_{i} x_{\pi(t)}$ for all $s, t \in\{1, \ldots, k\}$ and $i \in\{0, \ldots, T\}$. Consequently, to each $\mathcal{P}$ and $\pi$, there is associated a temporal decision profile ${ }^{\pi} \mathcal{P}=\left({ }^{\pi} \mathcal{R}_{0}, \ldots,{ }^{\pi} \mathcal{R}_{T}\right)$.

Likewise, any permutation $\sigma$ of the moments of time $\left\{t_{0}, \ldots, t_{T}\right\}$ determines a permutation of $\mathcal{P}$ by $\mathcal{P}^{\sigma}=\left(\mathcal{R}_{\sigma(0)}, \ldots, \mathcal{R}_{\sigma(T)}\right)$.

For each temporal decision profile $\mathcal{P} \in \mathbf{P}(\mathbf{X})$, its restriction to subprofile on the time in $I \subseteq \mathbf{T}$ (here $I$ is a subset of $\mathbf{T}$ that contains consecutive elements of $\mathbf{T}$ ), denoted $\mathcal{P}^{I}$, namely temporal decision subprofile, arises from exactly selecting the temporal decisions of $\mathcal{P}$ that are associated with the respective moments of time in $I$ (in the same order). To shorten notation, $\mathcal{P}^{i}$ is written instead of $\mathcal{P}^{I}$ when $I=\{i\}$. A overlapped partition is a finite collection of partitions $\left\{I_{1}, \ldots, I_{s}\right\}$ of $\mathbf{T}=\left\{t_{0}, \ldots, t_{T}\right\}$ satisfying:

- $I_{1} \cup \ldots \cup I_{s}=\mathbf{T}$

- For all $i \neq j,\left|I_{i} \cap I_{j}\right|= \begin{cases}1 & \text { if }|i-j|=1 \text {; } \\ 0 & \text { otherwise. }\end{cases}$

The time sequence that integrates the $k$-th element of the overlapped partition is labelled by $I_{k}=\left\{t_{0}^{I_{k}}, \ldots, t_{T_{k}}^{I_{k}}\right\}$, and therefore $\left|I_{k}\right|=T_{k}+1$. Taking into account this notation, the previous conditions imply:

- $T_{1}+T_{2}+\ldots+T_{s}=T$

- $t_{T_{k}}^{I_{k}}=t_{0}^{I_{k+1}}, \quad$ for $k=1, \ldots, s-1$.

Each overlapped partition generates a decomposition of $\mathcal{P}$ into consecutive temporal decision subprofiles $\mathcal{P}^{I_{1}}, \mathcal{P}^{I_{2}}, \ldots, \mathcal{P}^{I_{s}}$ such that $\mathcal{P}^{I_{1} \cup I_{2} \cup \ldots \cup I_{s}}=\mathcal{P}$.

Let $\mathbf{T}=\left\{t_{0}, \ldots, t_{T}\right\}$ be a temporal set of $T+1$ consecutive moments of time and suppose that $q$ new consecutive times are added to $\mathbf{T}$, in the following way 
$\overline{\mathbf{T}}^{q}=\left\{t_{0}, \ldots, t_{T}, t_{T+1}, \ldots, t_{T+q}\right\}$. The set $\overline{\mathbf{T}}^{q}$ is called extended temporal set. An temporal extension of a temporal decision profile $\mathcal{P} \in \mathbf{P}(\mathbf{X})$ is a new profile, $\overline{\mathcal{P}}^{q}$ such that includes $q$ new temporal decisions associated to the moments of time added to $\mathbf{T}$

$$
\overline{\mathcal{P}}^{q}=\left(\mathcal{R}_{0}, \ldots, \mathcal{R}_{T}, \mathcal{R}_{T+1}, \ldots, \mathcal{R}_{T+q}\right) \in \bigcup_{T \geq 1} \mathbf{W}(\mathbf{X})^{T+1+q}
$$

On occasion and for simplicity of notation, $\overline{\mathcal{P}}$ is written instead of $\overline{\mathcal{P}}^{1}$.

Let $\mathbf{X}=\left\{x_{1}, \ldots, x_{k}\right\}$ a finite set of $k$ alternatives and suppose that $q$ new alternatives are added to $\mathbf{X}$, such that $\widetilde{\mathbf{X}}=\left\{x_{1}, \ldots, x_{k}, x_{k+1}, \ldots, x_{k+q}\right\}$ denotes the expanded set of alternatives. The set of all complete preorders on $\widetilde{\mathbf{X}}$ is denoted by $\mathbf{W}(\widetilde{\mathbf{X}})$. Let $\widetilde{\mathcal{R}}_{i} \in \mathbf{W}(\widetilde{\mathbf{X}})$, it is called a expanded temporal decision of $\mathcal{R}_{i} \in$ $\mathbf{W}(\mathbf{X})$, if both temporal decisions grade the alternatives of set $\mathbf{X}$ in the same way, i.e $x_{r} \mathcal{R}_{i} x_{s} \Leftrightarrow x_{r} \widetilde{\mathcal{R}}_{i} x_{s}, \forall x_{r}, x_{s} \in \mathbf{X}$. An expanded temporal profile $\widetilde{\mathcal{P}}=$ $\left(\widetilde{\mathcal{R}}_{0}, \widetilde{\mathcal{R}}_{1}, \ldots, \widetilde{\mathcal{R}}_{T}\right)$ of $\mathcal{P}=\left(\mathcal{R}_{0}, \mathcal{R}_{1}, \ldots, \mathcal{R}_{T}\right)$ is a new temporal decision profile, such that each $\widetilde{\mathcal{R}_{i}}$ is an expanded temporal decision of $\mathcal{R}_{i}$.

Note that besides time, there exits different elements that can cause changes in preferences (for example, different choice order of alternatives, different scale of participants (social preferences), and so on). For short, in this paper the stability of preferences is only considered over time, but the study could be easily extended to other contexts.

Additionally, the reader should bear in mind that for simplicity this contribution does not distinguish between individual and social preferences and also it does not consider bounded rationality. These questions restrict the study due to the fact that over time the interaction between different individuals will lead to great differences between individual preferences and social preferences, specially 
when the bounded rationality of consumer behaviour is considered (see [28] and [29]). Thereby, the measuring models may be different for individual preferences stability and social preferences stability ${ }^{7}$.

In order to get the purpose of this research and to measure the stability of decisions over time, it is necessary to choose a strong codification procedure that allows to compare complete preorders at different moments of time without loss of preference consistency. Due to this fact, the procedure provided by GonzálezArtega, Alcantud and de Andrés Calle in [30], the canonical codification, is used in this work.

Given a a temporal decision $\mathcal{R}_{i} \in \mathbf{W}(\mathbf{X}), i \in\{0, \ldots, T\}$, its corresponding canonical codification is a real vector namely, the canonical codified temporal decision, defined by $\mathbf{c}_{\mathcal{R}_{i}}=\left(c_{1}^{\mathcal{R}_{i}}, \ldots, c_{k}^{\mathcal{R}_{i}}\right) \in(\{1, \ldots, k\})^{k}$, being $c_{j}^{\mathcal{R}_{i}}$ the number of alternatives classifying at most as good as the alternative $x_{j}$ at the moment of time $t_{i}$, i.e., $c_{j}^{\mathcal{R}_{i}}=\left|\left\{q: x_{j} \mathcal{R}_{i} x_{q}\right\}\right|$. The set of all possible canonical codified temporal decisions associated to $\mathbf{W}(\mathbf{X})$ is denoted by $\mathbf{F}=\mathbf{F}(\mathbf{W}(\mathbf{X}))$.

Given a temporal decision profile $\mathcal{P} \in \mathbf{P}(\mathbf{X})$, its corresponding canonical codified temporal decision profile is a $(T+1) \times k$ real matrix given by

$$
\mathcal{M}_{\mathcal{P}}=\left(\mathbf{c}_{\mathcal{R}_{0}}, \ldots, \mathbf{c}_{\mathcal{R}_{T}}\right)=\left(\begin{array}{ccc}
c_{1}^{\mathcal{R}_{0}} & \cdots & c_{k}^{\mathcal{R}_{0}} \\
\vdots & \ddots & \vdots \\
c_{1}^{\mathcal{R}_{T}} & \cdots & c_{k}^{\mathcal{R}_{T}}
\end{array}\right)
$$

where the row $i$-th indicates the canonical codified temporal decision $\mathbf{c}_{\mathcal{R}_{i}}$ at the moment of time $t_{i} \in \mathbf{T}$. Let $\mathbf{M}$ denote the set of all $(T+1) \times k$ real matrices ${ }^{8}$.

\footnotetext{
${ }^{7}$ This question will be addressed in future research.

${ }^{8}$ Example 1 includes a brief illustrative example of the notation and the canonical codification procedure.
} 
Any permutation $\pi$ of the alternatives $\{1, \ldots, k\}$ determines a canonical codified temporal decision profile ${ }^{\pi} \mathcal{M}_{\mathcal{P}} \in \mathrm{M}$ by permutation of the columns of $\mathcal{M}_{\mathcal{P}}$, such that, column $j$ of the profile ${ }^{\pi} \mathcal{M}_{\mathcal{P}}$ is column $\pi(j)$ of the codification profile $\mathcal{M}_{\mathcal{P}}$. Similarly, any permutation $\sigma$ of the moments of time $\left\{t_{0}, \ldots, t_{T}\right\}$ determines a canonical codified temporal decision profile $\mathcal{M}_{\mathcal{P}}^{\sigma}=\left(\mathbf{c}_{\mathcal{R}_{\sigma(0)}}, \ldots, \mathbf{c}_{\mathcal{R}_{\sigma(T)}}\right) \in$ $\mathrm{M}$ by permutation of the rows of $\mathcal{M}_{\mathcal{P}}$, such that, row $i$ of the profile $\mathcal{M}_{\mathcal{P}}^{\sigma}$ is row $\sigma(i)$ of the profile $\mathcal{M}_{\mathcal{P}}$.

\section{Decision stability measure: A novel approach}

So far as one can tell from today's research standpoint, there is not any theoretical definition of a measurement for establishing how much stable a decision is over time. For this reason, the findings of this work should make an important contribution to the field. The following section moves on to describe in greater detail our general proposal to measure decisions stability over time. Moreover, two particular definitions are established: the local and the global decision stability measures.

Definition 1. Let $\mathcal{P} \in \mathbf{P}(\mathbf{X})$ be a temporal decision profile. A decision stability measure over time is a mapping $\mu: \mathbf{P}(\mathbf{X}) \longrightarrow[0,1]$ that assigns to any temporal decision profile a value in the unit interval and it is defined imposing the following conditions:

(i) Full decision stability: $\mu(\mathcal{P})=1 \Longleftrightarrow \mathcal{R}_{0}=\ldots=\mathcal{R}_{T}$. In other words, $\mu(\mathcal{P})=1$ if and only if the temporal decision profile is constant over time.

(ii) Decision stability neutrality: $\mu\left({ }^{\pi} \mathcal{P}\right)=\mu(\mathcal{P})$ for each permutation $\pi$ of the alternatives, i.e., the alternatives rank does not change the stability of the 
decision.

Remark 1. Let $\mu$ be a decision stability measure, the corresponding decision instability measure is defined by $1-\mu$.

Definition 2. Let $\mathcal{P}=\left(\mathcal{P}_{0}, \ldots, \mathcal{P}_{T}\right) \in \mathbf{P}(\mathbf{X})$ be a temporal decision profile and its canonical codified profile $\mathcal{M}_{\mathcal{P}}=\left(\mathbf{c}_{\mathcal{R}_{0}}, \ldots, \mathbf{c}_{\mathcal{R}_{T}}\right) \in \mathbf{M}$. The local decision stability measure between the temporal decisions at the moments of time $t_{i-1}$ and $t_{i}$ is a mapping $\theta_{i}: \mathbf{W}(\mathbf{X}) \longrightarrow[0,1]$ given by

$$
\theta_{i}(\mathcal{P})=\theta_{[i-1, i]}(\mathcal{P})=1-\frac{\left\|\mathbf{c}_{\mathcal{R}_{i-1}}-\mathbf{c}_{\mathcal{R}_{i}}\right\|_{1}}{r}
$$

where $\mathbf{c}_{\mathcal{R}_{i-1}}$ and $\mathbf{c}_{\mathcal{R}_{i}}$ are the canonical codified vectors associated to the temporal decisions $\mathcal{R}_{i-1}$ and $\mathcal{R}_{i}$, respectively; $\|\cdot\|_{1}$ denotes the $l_{1}$-norm, then

$\left\|\mathbf{c}_{\mathcal{R}_{i-1}}-\mathbf{c}_{\mathcal{R}_{i}}\right\|_{1}=\sum_{h=1}^{k}\left|c_{h}^{\mathcal{R}_{i-1}}-c_{h}^{\mathcal{R}_{i}}\right| ;$ and finally, $r=\max _{\mathbf{c}, \mathbf{c}^{\prime} \in \mathbf{F}}\left\|\mathbf{c}-\mathbf{c}^{\prime}\right\|_{1}$. Therefore:

$$
\theta_{i}(\mathcal{P})=\theta_{[i-1, i]}(\mathcal{P})=1-\frac{\sum_{h=1}^{k}\left|c_{h}^{\mathcal{R}_{i-1}}-c_{h}^{\mathcal{R}_{i}}\right|}{\max _{\mathbf{c}, \mathbf{c}^{\prime} \in \mathbf{F}}\left\|\mathbf{c}-\mathbf{c}^{\prime}\right\|_{1}}
$$

In words, the local decision stability measure captures the stability between two consecutive temporal decisions as one minus the proportion of the number of preferences changes over the maximum that could happen between such two consecutive moments of time.

Lemma 1. Every local decision stability measure is a decision stability measure. 
Proposition 1. Let $\mathcal{R}_{i}, \mathcal{R}_{j} \in \mathbf{W}(\mathbf{X})$ be two two temporal decisions. The maximum instability between two temporal decisions is the diameter of the convex hull of the set of all canonical codifications associated to $\mathbf{W}(\mathbf{X})$, i.e., $\mathbf{F}=\mathbf{F}(\mathbf{W}(\mathbf{X}))$ for the $l_{1}$-norm. Formally,

$$
r=\max _{u, v \in \operatorname{Conv}(\boldsymbol{F})}\|u-v\|_{1}
$$

where $\operatorname{Conv}(\boldsymbol{F})=\left\{\sum_{j=1}^{|\boldsymbol{F}|} \alpha_{j} \cdot \boldsymbol{c}_{j}: 0 \leq \alpha_{j} \leq 1, \sum_{j=1}^{|\boldsymbol{F}|} \alpha_{j}=1\right\}$.

Proposition 2. The maximum instability between two temporal decisions, $r$, is determined by the following expressions:

- For an even number of alternatives, that is, for $\mathbf{X}=\{1, \ldots, k\}$ with even $k$

$$
r=\frac{3}{4} k^{2}-\frac{k}{2}
$$

- For an odd number of alternatives, that is, for $\mathbf{X}=\{1, \ldots, k\}$ with odd $k$

$$
r=\frac{3}{4} k^{2}-\frac{k}{2}-\frac{1}{4}
$$

Definition 3. Let $\mathcal{P} \in \mathbf{P}(\mathbf{X})$ a decision temporal profile and $\mathcal{M}_{\mathcal{P}} \in \mathbf{M}$ its corresponding canonical codification matrix. The global decision stability measure for the temporal decision profile $\mathcal{P} \in \mathbf{P}(\mathbf{X})$ is the mapping $\Theta: \mathbf{P}(\mathbf{X}) \times \mathbb{R}^{+} \longrightarrow[0,1]$ given by

$$
\Theta(\mathcal{P}, \lambda)=\sum_{i=1}^{T} w_{i, T}(\lambda) \cdot \theta_{i}(\mathcal{P})
$$

where

$$
w_{i, T}(\lambda)=A_{T}(\lambda) \cdot e^{-\lambda(T-i)} \quad \text { and } \quad A_{T}(\lambda)= \begin{cases}\frac{1-e^{-\lambda}}{1-e^{-\lambda T}} & \text { if } \quad \lambda>0 \\ \frac{1}{T} & \text { if } \quad \lambda=0\end{cases}
$$


Remark 2. Note that $\sum_{i=1}^{T} w_{i, T}(\lambda)=1$, for any $\lambda \in \mathbb{R}^{+}$.

This measure is the $\lambda$-weighted mean of the local decision stability measures over time.

Lemma 2. Every global decision stability measure is a decision stability measure.

The main points to emphasize in Definition 3 are the role of the local decision stability measures weights and the role of the $\lambda$-parameter. As far as the first question is concerned, the weights of the local decision stability measures depend on the number of the moments of time included in the decision temporal profile and consequently, they are different for each $T$.

With regard to the second question, the $\lambda$-parameter depicts the loss memory effect on decision over time. In this sense:

- For $\lambda>0$, a decision is taken on the set of alternatives $\mathbf{X}$ at the moment of time $t_{i}$ but taking into account more intensively the most recent temporal decisions.

- For $\lambda=0$, a decision is taken on the set of alternatives $\mathbf{X}$ at the moment of time $t_{i}$ but taking equally into account the previous temporal decisions.

Proposition 3. The global decision stability measure for a given decision temporal profile is a continuous function on $\lambda$-parameter.

Remark 3. Given a temporal decision profile $\mathcal{P} \in \mathbf{P}(\mathbf{X})$, the limit of the global decision stability measure for $\mathcal{P}$ as $\lambda$ approaches $\infty$ is the value of the local 
decision stability measure between the temporal decisions $T-1$ and $T, \theta_{T}(\mathcal{P})$. That is,

$$
\lim _{\lambda \rightarrow \infty} \Theta(\mathcal{P}, \lambda)=\theta_{T}(\mathcal{P})
$$

Now, an example is introduced for improving understanding and strengthening the basic concepts described above.

Example 1. Following the example that was presented in Introduction (ranking of daily menu starters), the set of alternatives is given by $\mathrm{X}=$ \{roast vegetarian, seafood chowder, caesar salad, stag's grilled $\}$, the temporal set, $\mathbf{T}=\left\{t_{0}, t_{1}, t_{3}\right\}$, includes three consecutive time steps and there are three customers $\mathbf{N}=\{1,2,3\}$, i.e., there are three temporal decision profiles: $\mathcal{P}^{1}, \mathcal{P}^{2}$ and $\mathcal{P}^{3}$ (one for every customer). The corresponding canonical codified temporal decision profiles are $\mathcal{M}_{\mathcal{P}^{1}}, \mathcal{M}_{\mathcal{P}^{2}}$ and $\mathcal{M}_{\mathcal{P}^{3}}$.

To analyse the level of stability of customers' preferences over time, the local and the global decision stability measures provided by Definitions 2 and 3 are used. To do so, first it is necessary to compute the diameter of the convex hull of the corresponding $\mathbf{F}$ being $k=4$ (see Proposition 1):

$$
r=\frac{3}{4} k^{2}-\frac{k}{2}=10
$$

and second, to compute the local stability measure for each customer:

$$
\mathcal{M}_{\mathcal{P}^{1}}=\left(\mathbf{c}_{\mathcal{R}_{0}^{1}}, \mathbf{c}_{\mathcal{R}_{1}^{1}}\right)=\left(\begin{array}{llll}
4 & 3 & 2 & 2 \\
4 & 3 & 2 & 2 \\
3 & 4 & 2 & 2
\end{array}\right) \Rightarrow\left\{\begin{array}{l}
\theta_{1}\left(\mathcal{P}^{1}\right)=1-\frac{0+0+0+0}{10}=1 \\
\theta_{2}\left(\mathcal{P}^{1}\right)=1-\frac{1+1+0+0}{10}=0.8
\end{array}\right.
$$




$$
\begin{aligned}
& \mathcal{M}_{\mathcal{P}^{2}}=\left(\mathbf{c}_{\mathcal{R}_{0}^{2}}, \mathbf{c}_{\mathcal{R}_{1}^{2}}\right)=\left(\begin{array}{llll}
1 & 2 & 3 & 4 \\
2 & 3 & 4 & 1 \\
2 & 3 & 4 & 1
\end{array}\right) \Rightarrow\left\{\begin{array}{l}
\theta_{1}\left(\mathcal{P}^{2}\right)=1-\frac{1+1+1+3}{10}=0.4 \\
\theta_{2}\left(\mathcal{P}^{2}\right)=1-\frac{0+0+0+0}{10}=1
\end{array}\right. \\
& \mathcal{M}_{\mathcal{P}^{3}}=\left(\mathbf{c}_{\mathcal{R}_{0}^{3}}, \mathbf{c}_{\mathcal{R}_{1}^{3}}\right)=\left(\begin{array}{llll}
1 & 3 & 4 & 2 \\
4 & 3 & 2 & 1 \\
3 & 1 & 2 & 4
\end{array}\right) \Rightarrow\left\{\begin{array}{l}
\theta_{1}\left(\mathcal{P}^{3}\right)=1-\frac{3+0+2+1}{10}=0.4 \\
\theta_{2}\left(\mathcal{P}^{3}\right)=1-\frac{1+2+0+3}{10}=0.4
\end{array}\right.
\end{aligned}
$$

Intuitively, $\theta_{2}\left(\mathcal{P}^{1}\right)=0.8$ means that the number of changes between the temporal decisions $\mathcal{R}_{0}^{1}$ and $\mathcal{R}_{1}^{1}$ over the maximum that could happen (10) is 2 .

Finally, Table 3 reports the weight values of the local decision stability measure for $T=2$, for diferent values of $\lambda$-parameter. Table 4 shows the values of the global stability measures for the three customers analysed. As can be seen from Table 4, Customers 1 and 2 reported significantly more global stability measure than Customer 3 regardless of $\lambda$-parameter. Therefore, the manager team could change the daily menu taking into account preferences of Customers 1 and 2 whatever the period of time.

\begin{tabular}{crrrrr} 
& \multicolumn{6}{c}{$w_{i, T}(\lambda)$} \\
\cline { 3 - 6 }$i \backslash \lambda$ & 0 & 0.1 & 0.25 & 0.5 & 0.75 \\
& & & & & \\
\hline 1 & 0.50 & 0.47 & 0.44 & 0.38 & 0.32 \\
2 & 0.50 & 0.53 & 0.56 & 0.62 & 0.68 \\
\hline
\end{tabular}

Table 3: Weights values of the local decision stability measures for different values of $\lambda$-parameter.

\begin{tabular}{lrrrrr} 
& \multicolumn{6}{c}{$\lambda$} \\
\cline { 2 - 6 } & 0 & 0.1 & 0.25 & 0.5 & 0.75 \\
\hline$\Theta\left(\mathcal{P}^{1}, \lambda\right)$ & 0.90 & 0.89 & 0.89 & 0.88 & 0.86 \\
$\Theta\left(\mathcal{P}^{2}, \lambda\right)$ & 0.70 & 0.71 & 0.74 & 0.77 & 0.81 \\
$\Theta\left(\mathcal{P}^{3}, \lambda\right)$ & 0.40 & 0.40 & 0.40 & 0.40 & 0.40 \\
\hline
\end{tabular}

Table 4: Global stability measures for $\mathcal{P}^{1}, \mathcal{P}^{2}$ and $\mathcal{P}^{3}$ attending to several values of $\lambda$. 


\section{Global decision stability measure: Properties and behaviour}

The global decision stability measure verifies some meaningful characteristics and properties. This section describes and analyses them. Moreover, in-depth studies on monotonicity property and weights behaviour have been included.

Let $\mathcal{P} \in \mathbf{P}(\mathbf{X})$ be a temporal decision profile and $\mathcal{M}_{\mathcal{P}} \in \mathbf{M}$ its corresponding canonical codification matrix.

Compensativeness. This property ensures that the global decision stability measure is always defined between the minimum and the maximum value of the local decision stability measure. Formally,

$\min \left(\theta_{1}(\mathcal{P}), \ldots, \theta_{T}(\mathcal{P})\right) \leq \Theta(\mathcal{P}, \lambda) \leq \max \left(\theta_{1}(\mathcal{P}), \ldots, \theta_{T}(\mathcal{P})\right) \quad$ whenever $\lambda \geq 0$

Convexity. The computation of the global decision stability measure of a decision temporal profile on $k$ alternatives at $T+1$ moments of time is a weighted average of the corresponding measures of two consecutive temporal subprofiles. Formally,

Let $\mathcal{P} \in \mathbf{P}(\mathbf{X})$ be a temporal decision profile and $I_{1}, I_{2}$ an overlapped partition of $\mathbf{T}$, with

$$
I_{1}=\left\{t_{0}=t_{0}^{I_{1}}, \ldots, t_{T_{1}}^{I_{1}}\right\}, \quad I_{2}=\left\{t_{T_{1}}^{I_{1}}=t_{0}^{I_{2}}, \ldots, t_{T_{2}}^{I_{2}}=t_{T}\right\}, \quad T_{1}+T_{2}=T
$$

Then,

$$
\Theta(\mathcal{P}, \lambda)=\alpha_{\lambda, T}\left(I_{1}\right) \cdot \Theta\left(\mathcal{P}^{I_{1}}, \lambda\right)+\alpha_{\lambda, T}\left(I_{2}\right) \cdot \Theta\left(\mathcal{P}^{I_{2}}, \lambda\right),
$$

where the weights $\alpha_{\lambda, T}(\cdot)$ are defined by:

$\alpha_{\lambda, T}\left(I_{1}\right)=\left\{\begin{array}{ll}\frac{e^{-\lambda\left(T-T_{1}\right)}-e^{-\lambda T}}{1-e^{-\lambda T}} & \text { if } \lambda \neq 0 \\ \frac{T_{1}}{T} & \text { if } \lambda=0\end{array}, \quad \alpha_{\lambda, T}\left(I_{2}\right)= \begin{cases}\frac{1-e^{-\lambda\left(T-T_{1}\right)}}{1-e^{-\lambda T}} & \text { if } \lambda \neq 0 \\ \frac{T_{2}}{T} & \text { if } \lambda=0\end{cases}\right.$ 
Remark 4. If $I_{1}, I_{2}$ is a overlapped partition of $\mathbf{T}=\left\{t_{0}, t_{1}, \ldots, t_{T}\right\}$, then it is straightforward to check that: $\alpha_{\lambda, T}\left(I_{1}\right)+\alpha_{\lambda, T}\left(I_{2}\right)=1$.

The convexity property can be extended to the case of any collection of subprofiles generated by an overlapped partition of the temporal set T. As a consequence, the global decision stability measure of $\mathcal{P}$ can be built from the decision stability measures of such sub-profiles. This is addressed in Subsection 5.2.

No Timelessness. Only if $\lambda=0$ permutations on time moments do not change the stability of the decision over time. Formally,

$$
\Theta\left(\mathcal{P}^{\sigma}, \lambda\right)=\Theta(\mathcal{P}, \lambda)
$$

Convergence to full stability. Suppose that $q$ new consecutive times are added to $\mathbf{T}, \overline{\mathbf{T}}^{q}=\left\{t_{0}, \ldots, t_{T}, t_{T+1}, \ldots, t_{T+q}\right\}$ and that the temporal extension of the temporal decision profile $\mathcal{P} \in \mathbf{P}(\mathbf{X}), \quad \overline{\mathcal{P}}^{q}$ is defined such that $\mathcal{R}_{T+1}=\ldots=\mathcal{R}_{T+q}$ then the global decision stability measure of $\overline{\mathcal{P}}^{q}$ approaches one as $q$ approaches infinity. Formally,

$$
\lim _{q \rightarrow \infty} \Theta\left(\overline{\mathcal{P}}^{q}, \lambda\right)=1, \quad \text { for all } \lambda \geq 0
$$

\subsection{Discussion on Monotonicity}

For the purpose of analysis of the consistency of the global decision stability measure and taking into account the crucial importance of the monotonicity property in many everyday temporal decisions, the following part of this paper moves on to describe in greater detail the behaviour of the global decision stability measure under several monotonicity approaches. 
Absolute monotonicity. Suppose that a new moment of time $t_{T+1}$ is added to $\mathbf{T}$, and a temporal decision is taken on $\mathbf{X}$ at $t_{T+1}$ in the sense $\mathcal{R}_{T}=\mathcal{R}_{T+1}$. If $\mathcal{P}$ is not constant over time, then the global decision stability measure of the enlarged profile $\overline{\mathcal{P}}$ is strictly higher than the original one. Formally,

$$
\Theta(\mathcal{P}, \lambda)<\Theta(\overline{\mathcal{P}}, \lambda) \quad \text { if } \Theta(\mathcal{P}, \lambda)<1
$$

Remark 5. In the same way as absolute monotonicity property, suppose that a new moment of time $t_{T+1}$ is added to $\mathbf{T}$, but now a temporal decision is taken on $\mathbf{X}$ at $t_{T+1}$ in the sense that the temporal decision makes in $T+1$, i.e., $\mathcal{R}_{T+1}$ is opposite to the temporal decision made in $T, \mathcal{R}_{T}$. If $\mathcal{P}$ is not constant over time, then the global decision stability measure of the enlarged profile $\overline{\mathcal{P}}$ is strictly smaller than the original one. Formally,

$$
\begin{aligned}
& \text { If } \mathcal{R}_{T} \neq \mathcal{R}_{T+1} \text { and }\left\|\mathbf{c}_{\mathcal{R}_{T}}-\mathbf{c}_{\mathcal{R}_{T+1}}\right\|_{1}=\max _{\mathbf{c}, \mathbf{c}^{\prime} \in \mathbf{F}}\left\|\mathbf{c}-\mathbf{c}^{\prime}\right\|_{1}=r \text {, then } \\
& \theta_{T+1}\left(\overline{\mathcal{P}}^{I_{2}}\right)=0 \text {. Consequently, } \\
& \Theta(\mathcal{P}, \lambda)>\Theta(\overline{\mathcal{P}}, \lambda) \text { and } \Theta(\overline{\mathcal{P}}, \lambda) \in[0, \Theta(\mathcal{P}, \lambda)) .
\end{aligned}
$$

Relative monotonicity. Suppose that a new moment of time $t_{T+1}$ is added to $\mathbf{T}$, and a temporal decision is taken on $\mathbf{X}$ at $t_{T+1}$ in the sense $\mathcal{R}_{T} \neq \mathcal{R}_{T+1}$ but $r>\left\|\mathbf{c}_{\mathcal{R}_{T}}-\mathbf{c}_{\mathcal{R}_{T+1}}\right\|_{1}>0$, i.e., the temporal decisions at $T$ and $T+1$ are different but not opposite. If $\mathcal{P}$ is not constant over time, then the global decision stability measure of the enlarged profile $\overline{\mathcal{P}}$ is strictly higher than the minimun of the stabilities of the original one and the subprofile generated by the two last time steps. Formally,

$$
\Theta(\overline{\mathcal{P}}, \lambda) \geq \min \left\{\Theta(\mathcal{P}, \lambda), \Theta\left(\overline{\mathcal{P}}^{\left\{t_{T}, t_{T+1}\right\}}, \lambda\right)\right\}
$$


Comparative monotonicity. Let $\overline{\mathbf{T}}=\left\{t_{0}, \ldots, t_{T}, t_{T+1}\right\}$ be an extended temporal set. Let $\overline{\mathcal{P}}, \overline{\mathcal{P}}^{\prime} \in \bigcup_{T \geq 1} \mathbf{W}(\mathbf{X})^{T+2}$ be two extended temporal decision profiles associated to $\overline{\mathbf{T}}$ such that $\overline{\mathcal{P}}=\left(\mathcal{R}_{0}, \ldots, \mathcal{R}_{T}, \mathcal{R}_{T+1}\right)$ and $\overline{\mathcal{P}}^{\prime}=\left(\mathcal{R}_{0}, \ldots, \mathcal{R}_{T}, \mathcal{R}_{T+1}^{\prime}\right)$. If the relationship between $\mathcal{R}_{T+1}$ and $\mathcal{R}_{T+1}^{\prime}$ is given by

$$
\left\|\mathbf{c}_{\mathcal{R}_{T}}-\mathbf{c}_{\mathcal{R}_{T+1}^{\prime}}\right\|_{1}>\left\|\mathbf{c}_{\mathcal{R}_{T}}-\mathbf{c}_{\mathcal{R}_{T+1}}\right\|_{1}
$$

then, the global decision stability measure of the enlarged profile $\overline{\mathcal{P}}$ is strictly higher than the global decision stability measure of the enlarged profile $\overline{\mathcal{P}}^{\prime}$. Formally,

$$
\Theta(\overline{\mathcal{P}}, \lambda)>\Theta\left(\overline{\mathcal{P}}^{\prime}, \lambda\right)
$$

Decision monotonicity. Let $\widetilde{\mathbf{X}}=\left\{x_{1}, \ldots, x_{k}, x_{k+1}, \ldots, x_{k+q}\right\}$ be an expanded set of alternatives of $\mathbf{X}$. Let $\mathcal{P}=\left(\mathcal{R}_{1}, \ldots, \mathcal{R}_{T}\right)$ a profile, and $\widetilde{\mathcal{P}}=\left(\widetilde{\mathcal{R}}_{1}, \ldots, \widetilde{\mathcal{R}}_{T}\right)$ any expanded temporal profile such that:

1. The temporal decision on the set of new alternatives is constant over time, that is, $\left.\widetilde{\mathcal{R}}_{i}\right|_{\left\{x_{k+1}, \ldots, x_{k+q}\right\}}=\left.\widetilde{\mathcal{R}}_{j}\right|_{\left\{x_{k+1}, \ldots, x_{k+q}\right\}}$, for all $i, j \in\{0, \ldots, T\}$.

2. The new alternatives are always better or worse graded that old ones, that is, $x_{r} \widetilde{\mathcal{R}}_{i} x_{s}$ or $x_{s} \widetilde{\mathcal{R}}_{i} x_{r}$ for all $r \in\{1, \ldots, k\}, s \in\{k+1, \ldots, k+q\}$, and $i \in\{0, \ldots, T\}$. Then, the global decision stability measure of the expanded profile $\widetilde{\mathcal{P}}$ is strictly higher than the original one. Formally:

$$
\Theta(\mathcal{P}, \lambda)<\Theta(\widetilde{\mathcal{P}}, \lambda)
$$

\subsection{Weights behaviour: Decomposition of the global stability decision measure}

Weights behaviour is an essential aspect of study in decision making problems due to their changes can greatly affect results. Therefore, it is necessary to know how weights can perform when changes in assumptions take place. 
On the basis of the above, this subsection is focused on analysing weights behaviour and then, how the global stability decision measure can be decomposed. For this propose, the convexity property is extended to the case of any collection of sub-profiles generated by an overlapped partition of the temporal set T. As a consequence, the global decision stability measure of $\mathcal{P}$ can be built from the decision stability measures of such sub-profiles. More specifically, the global decision stability measure of a temporal decision profile $\mathcal{P}$ is a weighted average of the corresponding global decision stability measures in each sub-profile where the weights represent the contributions of the different sub-profiles to the global decision stability measure. The following proposition ensures the aforementioned issues.

Proposition 4. Let $\mathcal{P} \in \mathbf{P}(X)$ be a temporal decision profile on the time sequence $\mathbf{T}=\left\{t_{0}, \ldots, t_{T}\right\}$ and $\left\{I_{1}, \ldots, I_{s}\right\}$ an overlapped partition of $\mathbf{T}$, such that $I_{k}=\left\{t_{0}^{I_{k}}, \ldots, t_{T_{k}}^{I_{k}}\right\}$ for $k=1, \ldots, s$. Then

$$
\Theta(\mathcal{P}, \lambda)=\sum_{k=1}^{s} \alpha_{\lambda, T}\left(I_{k}\right) \cdot \Theta\left(\mathcal{P}^{I_{k}}, \lambda\right)
$$

where $\alpha_{\lambda, T}(\cdot)$ is given by

$$
\alpha_{\lambda, T}\left(I_{k}\right)= \begin{cases}\frac{e^{-\lambda\left(T-T_{k}^{+}\right)}-e^{-\lambda\left(T-T_{k}^{-}\right)}}{1-e^{-\lambda T}} & \text { if } \lambda \neq 0 \\ \frac{T_{k}}{T} & \text { if } \lambda=0\end{cases}
$$

being $T_{k}^{+}=\sum_{j=1}^{k} T_{j}$ and $T_{k}^{-}=\sum_{j=1}^{k-1} T_{j}$ the upper and the lower times indices of the subprofile $I_{k}$, respectively and it satisfies $\sum_{k=1}^{s} \alpha_{\lambda, T}\left(I_{k}\right)=1$. 


\section{Discussion and real examples}

In the following pages, it is going to put in practice the methodologies proposed in Section 4. To this purpose two cases of study are presented. The first one explores into the characteristics of Spanish citizens' voting behaviour. The second one attempts to evaluate European citizens' preferences about passenger cars by means of a particular survey: a comparative research including preferences from three different European countries: Germany, Norway and Spain.

\subsection{The stability of the political opinion in the Spanish society: A case of study}

The objective of this case of study is to research into the characteristics of Spanish citizens' voting behaviour and also to measure the stability of such voting opinions.

Data for this study were collected from the CIS, the Spanish Center of Sociological Research. The CIS carries out polls on a monthly basis and they include interviews from around 2,500 randomly-chosen Spanish people ${ }^{9}$ although polls only contain questions on voting intention in January, April, July and October ${ }^{10}$. In particular, this research incorporates the polls from January 2016 to October 2018 , i.e., twelve moments of time that can be represented by the temporal set, $\mathbf{T}=\left\{t_{0}, \ldots, t_{11}\right\}$ where $t_{0}$ corresponds to January 2016, $t_{1}$ to April 2016, $t_{2}$ to July 2016 and so on, up to $t_{11}$ that corresponds to October 2018. During theses moments of time the set of candidates is made up of four political parties: Partido

\footnotetext{
${ }^{9}$ All data are available though the website: http://www.cis.es/cis/opencm/EN/ 11_barometros/index.jsp

${ }^{10}$ Apart from people's political preferences, interviews incorporate social and demographic information.
} 
Popular (PP), Partido Socialista Obrero Español (PSOE), Grupo Podemos (GP) and Ciudadanos (C's) that it can be denoted by $\mathbf{X}=\left\{P P, P S O E, G P, C^{\prime} s\right\}$.

The Spanish society by means of the aforementioned voting opinions surveys establishes the temporal decisions ${ }^{11}$ on the set of candidates $\mathbf{X}$ at $\mathbf{T}$. Such temporal decisions are collected in Table 5.

\begin{tabular}{|c|c|c|}
\hline 2016 & 2017 & 2018 \\
\hline Jun. $\quad \mathcal{R}_{0}: G P \succ P P \succ P S O E \succ C^{\prime} s$ & Jun. $\quad \mathcal{R}_{4}: P P \succ G P \succ P S O E \succ C^{\prime} s$ & Jun. $\quad \mathcal{R}_{8}: P S O E \succ C^{\prime} s \succ P P \succ G P$ \\
\hline Apr. $\quad \mathcal{R}_{1}: G P \succ P P \succ P S O E \succ C^{\prime} s$ & Apr. $\quad \mathcal{R}_{5}: P P \succ G P \succ P S O E \succ C^{\prime} s$ & Apr. $\quad \mathcal{R}_{9}: C^{\prime} s \succ P S O E \succ P P \succ G P$ \\
\hline Jul. $\quad \mathcal{R}_{2}: P P \succ P S O E \succ G P \succ C^{\prime} s$ & Jul. $\quad \mathcal{R}_{6}: P S O E \succ P P \succ G P \succ C^{\prime} s$ & Jul. $\quad \mathcal{R}_{10}: P S O E \succ C^{\prime} s \succ P P \succ G P$ \\
\hline Oct. $\quad \mathcal{R}_{3}: P P \succ G P \succ P S O E \succ C^{\prime} s$ & Oct. $\quad \mathcal{R}_{7}: P P \succ P S O E \succ C^{\prime} s \succ G P$ & Oct. $\quad \mathcal{R}_{11}: P S O E \succ C^{\prime} s \succ P P \succ G P$ \\
\hline
\end{tabular}

Table 5: Temporal decisions established by voting opinions surveys.

From temporal decisions on Table 5, the following temporal decision profile is obtained: $\mathcal{P}=\left(\mathcal{R}_{0}, \mathcal{R}_{1}, \mathcal{R}_{2}, \mathcal{R}_{3}, \mathcal{R}_{4}, \mathcal{R}_{5}, \mathcal{R}_{6}, \mathcal{R}_{7}, \mathcal{R}_{8}, \mathcal{R}_{9}, \mathcal{R}_{10}, \mathcal{R}_{11}\right)$ and its corresponding canonical codified temporal decision profile is given by $\mathcal{M}_{\mathcal{P}}=$ $\left(\mathbf{c}_{\mathcal{R}_{0}}, \ldots, \mathbf{c}_{\mathcal{R}_{10}}, \mathbf{c}_{\mathcal{R}_{11}}\right)$.

Once the framework of the decision making problem has been established, the local decision stability measures can be computed by Definition 2. Table 6 collects such values.

\begin{tabular}{cccccccccccc} 
& \multicolumn{110}{c}{$i$} \\
\cline { 2 - 11 } & 1 & 2 & 3 & 4 & 5 & 6 & 7 & 8 & 9 & 10 & 11 \\
\hline$\theta_{i}(\mathcal{P})$ & 1.0 & 0.6 & 0.8 & 1.0 & 1.0 & 0.6 & 0.6 & 0.6 & 0.8 & 0.8 & 1.0 \\
\hline
\end{tabular}

Table 6: Values of local stability measures.

\footnotetext{
${ }^{11}$ Data from direct estimation.
} 
Now and in order to compute the global decision stability measure for $\mathcal{P}$ and to analyse the effect of the $\lambda$-parameter, i.e., the loss memory effect of the Spanish society on political parties over time, different values $\lambda$-parameter are considered specifically, $\lambda=\{0,0.1,0.25,0.5,0.75\}$. Taking into account such values of the $\lambda$-parameter, Table 7 shows the different weights values of the local decision stability measures $w_{i, T}(\lambda), i=1, \ldots, 11$ computed by means of Definition 3 .

\begin{tabular}{lrrrrrrrrrrrr} 
& \multicolumn{10}{c}{$w_{i, T}(\lambda)$} \\
\cline { 2 - 12 }$\lambda$ & 1 & 2 & 3 & 4 & 5 & 6 & 7 & 8 & 9 & 10 & 11 \\
\hline 0 & 0.0909 & 0.0909 & 0.0909 & 0.0909 & 0.0909 & 0.0909 & 0.0909 & 0.0909 & 0.0909 & 0.0909 & 0.0909 \\
0.1 & 0.0525 & 0.0580 & 0.0641 & 0.0708 & 0.0783 & 0.0865 & 0.0956 & 0.1057 & 0.1168 & 0.1291 & 0.1426 \\
0.25 & 0.0194 & 0.0249 & 0.0320 & 0.0411 & 0.0527 & 0.0677 & 0.0869 & 0.1116 & 0.1433 & 0.1840 & 0.2363 \\
0.5 & 0.0027 & 0.0044 & 0.0072 & 0.0119 & 0.0197 & 0.0324 & 0.0535 & 0.0882 & 0.1453 & 0.2396 & 0.3951 \\
0.75 & 0.0003 & 0.0006 & 0.0013 & 0.0028 & 0.0059 & 0.0124 & 0.0263 & 0.0556 & 0.1178 & 0.2493 & 0.5278 \\
\hline
\end{tabular}

Table 7: Weights values of the local decision stability measures, $w_{i, T}(\lambda)$, for different values of $\lambda$-parameter.

Finally, the global decision stability measures for the different values of $\lambda$ parameter are showed in Table 8. As can be seen from Table 5, Spanish voting

\begin{tabular}{rrrrrr} 
& \multicolumn{6}{c}{$\lambda$} \\
\cline { 2 - 6 } & 0 & 0.1 & 0.25 & 0.5 & 0.75 \\
\hline$\Theta(\mathcal{P}, \lambda)$ & 0.8000 & 0.7997 & 0.8117 & 0.8502 & 0.8884 \\
\hline
\end{tabular}

Table 8: Global stability measures for $\mathcal{P}$ attending to several values of $\lambda$.

decisions could seem uneven because some temporal decisions are exactly the opposite, for instance:

$$
\mathcal{R}_{0}: G P \succ P P \succ P S O E \succ C^{\prime} s, \quad \quad \mathcal{R}_{9}: C^{\prime} s \succ P S O E \succ P P \succ G P
$$


and also there is no an exact match between any temporal decision. Attending to these relevant facts, one could think that there should be tenuous stability among Spanish political preferences but surprisingly, the global stability measures during $\mathbf{T}$ for several values of $\lambda$ are close to 1 (see Table 8), i.e., the results show a high level of stability of the Spanish society's political opinion. This result could give the impression of being somewhat counter-intuitive however, closer inspection of Table 5 shows that the Spanish society's opinion has undergone no abrupt modifications from period to period and consequently, most of the local decision stability measures are near 1, as it can observe in Table 6.

\subsection{Buying a car: Have European changed their mind?}

Drawing upon the strands of this research on preference stability, this study attempts to evaluate European citizens' preferences about passenger cars and to study if they are changing their mind on buying electrical cars.

Right now, the purchase of electric cars has moved from an environmental issue to a serious proposition for drivers. The sales of electrical cars are still weak but there is an increase in them due to the European Union (EU) regulations on combustions cars scheduled for 2050. The data confirms that drivers' preferences are changing but how are they changing? Are their decisions stable? In order to find answers to these questions this subsection includes a comparative analysis of passenger car market on three EU countries.

Moving on now to consider three European countries, Germany, Norway and Spain. The purpose of this case of study is to mak comparisons among the citizens' car preferences of the three aforementioned countries. Data were collec- 
ted from ACEA, the European Automobile Manufacturers' Association ${ }^{12}$. Even though ACEA publishes monthly passenger car registration data ${ }^{13}$, this enquiry incorporates the quarterly change in registrations for passenger cars from the first quarter of 2017 to the third quarter of 2018, considering then, 7 moments of time (quarters in this case) that can be represented by the temporal set, $\mathbf{T}=$ $\left\{t_{0}, t_{1}, t_{2}, t_{3}, t_{4}, t_{5}, t_{6}\right\}$ where $t_{0}$ corresponds to the first quarter of $2017, t_{1}$ to the second quarter of $2017, t_{2}$ to the third quarter of 2017 , and so on, up to $t_{6}$ that corresponds to the third quarter of 2018.

Based on the data provided by ACEA the passengers cars registered during these periods of time can be classified like battery electric vehicles (BE), pluggedin hybrid electric vehicles (PHE), hybrid electric vehicles (HE), alternativelypowered vehicles, other than electric (AP), fuel vehicles (F) and diesel vehicles (D). Therefore, the set of alternatives can be denoted by $\mathbf{X}=\{B E, P H E, H E, A P, F, D\}$.

Table 9 gathers citizens' temporal car preferences for each quarter from Germany, Norway and Spain. These preferences are based on data provided by ACEA.

From the temporal decisions presented in Table 9, Table 10 collects the temporal decision profiles for each country as well as their corresponding canonical codified temporal decision profiles.

Thereupon by means of Definition 2, the local decision stability measures for each country are computed. Table 11 collects separately such values for each country.

\footnotetext{
${ }^{12}$ All data available through the website: https://www.acea.be/statistics/tag/ category/passenger-cars-registrations

${ }^{13}$ Except in the summertime.
} 


\begin{tabular}{|c|c|c|c|}
\hline & Germany & & Norway \\
\hline \multirow{4}{*}{2017} & $Q_{1} \quad \mathcal{R}_{0}^{G}: B E \succ P H E \succ H E \succ D \succ A P \succ F$ & \multirow{4}{*}{2017} & $Q_{1} \quad \mathcal{R}_{0}^{N}: A P \succ P H E \succ H E \succ B E \succ D \succ F$ \\
\hline & $Q_{2} \quad \mathcal{R}_{1}^{G}: P H E \succ F \succ H E \succ D \succ A P \succ B E$ & & $Q_{2} \quad \mathcal{R}_{1}^{N}: B E \succ D \succ F \succ H E \succ P H E \succ A P$ \\
\hline & $Q_{3} \quad \mathcal{R}_{2}^{G}: A P \succ B E \succ P H E \succ H E \succ F \succ D$ & & $Q_{3} \quad \mathcal{R}_{2}^{N}: A P \succ P H E \succ B E \succ H E \succ F \succ D$ \\
\hline & $Q_{4} \quad \mathcal{R}_{3}^{G}: A P \succ B E \succ F \succ P H E \succ H E \succ D$ & & $Q_{4} \quad \mathcal{R}_{3}^{N}: A P \succ B E \succ P H E \succ F \succ H E \succ D$ \\
\hline \multirow{4}{*}{2018} & $Q_{1} \quad \mathcal{R}_{4}^{G}: A P \succ H E \succ B E \succ D \succ F \succ P H E$ & \multirow{3}{*}{2018} & $Q_{1} \quad \mathcal{R}_{4}^{N}: B E \succ D \succ F \succ P H E \succ H E \succ A P$ \\
\hline & $Q_{2} \quad \mathcal{R}_{5}^{G}: A P \succ H E \succ F \succ D \succ P H E \succ B E$ & & $Q_{2} \quad \mathcal{R}_{5}^{N}: H E \succ P H E \succ F \succ D \succ B E \succ A P$ \\
\hline & $Q_{3} \quad \mathcal{R}_{6}^{G}: H E \succ P H E \succ B E \succ D \succ F \succ A P$ & & $Q_{3} \quad \mathcal{R}_{6}^{N}: B E \succ D \succ H E \succ F \succ P H E \succ A P$ \\
\hline & & Spain & \\
\hline \multirow{4}{*}{2017} & $Q_{1} \quad \mathcal{R}_{0}^{S}: A P \succ P H E \succ H E \succ D \succ F \succ B E$ & \multirow{4}{*}{2018} & $Q_{1} \quad \mathcal{R}_{4}^{S}: A P \succ H E \succ D \succ F \succ B E \succ P H E$ \\
\hline & $Q_{2} \quad \mathcal{R}_{1}^{S}: P H E \succ A P \succ F \succ B E \succ H E \succ D$ & & $Q_{2} \quad \mathcal{R}_{5}^{S}: A P \succ P H E \succ H E \succ F \succ D \succ B E$ \\
\hline & $Q_{3} \quad \mathcal{R}_{2}^{S}: B E \succ A P \succ P H E \succ H E \succ D \succ F$ & & $Q_{3} \quad \mathcal{R}_{6}^{S}: B E \succ A P \succ H E \succ D \succ P H E \succ F$ \\
\hline & $Q_{4} \quad \mathcal{R}_{3}^{S}: A P \succ B E \succ P H E \succ B E \succ H E \succ D$ & & \\
\hline
\end{tabular}

Table 9: Quarterly car preferences in Germany, Norway and Spain.

\begin{tabular}{|c|c|c|}
\hline Germany & Norway & Spain \\
\hline $\mathcal{P}^{G}=\left(\mathcal{R}_{0}^{G}, \ldots, \mathcal{R}_{6}^{G}\right)$ & $\mathcal{P}^{N}=\left(\mathcal{R}_{0}^{N}, \ldots, \mathcal{R}_{6}^{N}\right)$ & $\mathcal{P}^{S}=\left(\mathcal{R}_{0}^{S}, \ldots, \mathcal{R}_{6}^{S}\right)$ \\
\hline $\mathcal{M}_{\mathcal{P}^{G}}=\left(\mathbf{c}_{\mathcal{R}_{0}^{G}}, \ldots, \mathbf{c}_{\mathcal{R}_{6}^{G}}\right)$ & $\mathcal{M}_{\mathcal{P}^{N}}=\left(\mathbf{c}_{\mathcal{R}_{0}^{N}}, \ldots, \mathbf{c}_{\mathcal{R}_{6}^{N}}\right)$ & $\mathcal{M}_{\mathcal{P}^{S}}=\left(\mathbf{c}_{\mathcal{R}_{0}^{S}}, \ldots, \mathbf{c}_{\mathcal{R}_{6}^{S}}\right)$ \\
\hline
\end{tabular}

Table 10: Temporal decision profiles and their corresponding canonical codified temporal decision profiles for every country.

With the goal of calculating the global decision stability measure for each profile, i.e., for each country, several values of $\lambda$-parameter have been considered. The weights values of the local decision stability measures are shown in Table 12. These weights values are the same for calculating the global decision stability measure for $\mathcal{P}_{G}, \mathcal{P}_{N}$ and $\mathcal{P}_{S}$.

Last but not least, having defined the weights values of the local decision stability measures, it now moves on to compute the global decision stability measures 
for $\mathcal{P}^{G}, \mathcal{P}^{N}$ and $\mathcal{P}^{S}$ to different values of $\lambda$-parameter. The values are shown in Table 13.

\begin{tabular}{lrrrrrr} 
& 1 & 2 & 3 & 4 & 5 & 6 \\
\cline { 2 - 7 } & 0.58 & 0.33 & 0.83 & 0.58 & 0.75 & 0.42 \\
\hline$\theta_{i}\left(\mathcal{P}^{G}\right)$ & 0.5 & & & \\
$\theta_{i}\left(\mathcal{P}^{N}\right)$ & 0.25 & 0.33 & 0.83 & 0.50 & 0.50 & 0.50 \\
$\theta_{i}\left(\mathcal{P}^{S}\right)$ & 0.58 & 0.58 & 0.58 & 0.50 & 0.67 & 0.50 \\
\hline
\end{tabular}

Table 11: Values of local stability measures for each country.

\begin{tabular}{lrrrrrr} 
& \multicolumn{6}{c}{$w_{i, T}(\lambda)$} \\
\cline { 2 - 7 }$\lambda$ & 1 & 2 & 3 & 4 & 5 & 6 \\
\hline 0 & 0.17 & 0.17 & 0.17 & 0.17 & 0.17 & 0.17 \\
0.1 & 0.13 & 0.14 & 0.16 & 0.17 & 0.19 & 0.21 \\
0.25 & 0.08 & 0.10 & 0.13 & 0.17 & 0.22 & 0.28 \\
0.5 & 0.03 & 0.06 & 0.09 & 0.15 & 0.25 & 0.41 \\
0.75 & 0.01 & 0.03 & 0.06 & 0.12 & 0.25 & 0.53 \\
\hline
\end{tabular}

Table 12: Weights values of the local decision stability measures, $w_{i, T}(\lambda)$, for different values of $\lambda$-parameter to Germany, Norway and Spain.

\begin{tabular}{lrrrrr} 
& \multicolumn{6}{c}{$\lambda$} \\
\cline { 2 - 6 } & 0 & 0.1 & 0.25 & 0.5 & 0.75 \\
\hline$\Theta\left(\mathcal{P}^{G}, \lambda\right)$ & 0.58 & 0.58 & 0.58 & 0.57 & 0.54 \\
$\Theta\left(\mathcal{P}^{N}, \lambda\right)$ & 0.49 & 0.50 & 0.51 & 0.51 & 0.51 \\
$\Theta\left(\mathcal{P}^{S}, \lambda\right)$ & 0.57 & 0.56 & 0.56 & 0.56 & 0.55 \\
\hline
\end{tabular}

Table 13: Global stability measures for $\mathcal{P}^{G}, \mathcal{P}^{N}$ and $\mathcal{P}^{S}$ attending to $\lambda$.

Many customers consider buying electric cars as a real chance. Cars electric market is already a reality and the electric cars supply is increasingly numerous, more active and varied. These facts generate that a growing number of drivers consider electric cars a perfectly valid option. However, the requirement of a connection point at home and travel limitations (not to travel much longer distance than 200-300 km between charges) make big cities the natural domain of electric cars, against long-distance journeys at least with some freedom of movement. 
All these factors provoke continuous changing drivers' preferences as can be seen from Table 9 and more particularly in Germany and Norway due to Spanish electric cars market is less developed. The results of the global stability measures corroborate all the foregoing as Table 13 shows.

\section{Conclusions and future research}

The main goal of the current study has been to determine a general procedure to measure decisions stability over time under the assumption of complete pre-orders. To develop a deep and strong general measure, two minimum conditions have been required, full decision stability and decision stability neutrality. Moreover, this work contributes to existing knowledge of preferences behaviour by providing two particular decision stability measures, the local and the global decision stability measure. The study of such measures is completed including the main features of the novel approaches as well as several mathematical results on the proposed measures behaviour. Therefore, this research will prove useful in expanding our understanding of how decision change over time from a theoretical and practical perspective. Moreover, the present contribution includes two real cases of study to explore into the characteristics of Spanish citizens' voting behaviour and to attempt to analyse European citizens' preferences about passenger car market.

Finally, this research has thrown up many questions in need of further investigation for example, it must be interesting to develop a new measure being able of capturing preferences disparity between non consecutive moments of time and to define a novel measurement specially design for social preferences stability considering the bounded rationality of consumer behaviour. 


\section{Acknowledgements}

The authors thank the two anonymous reviewers and James R. Marsden (Editorin- Chief) for their valuable comments and recommendations. R. de Andrés Calle and T. González-Arteaga acknowledge financial support by the Spanish Ministerio de Economía y Competitividad under Project ECO2016-77900-P.

\section{References}

[1] J. Stigler, G. Becker, De gustibus non est disputandum, American Economic Review 2 (67) (1977) 76-90.

[2] Y. Chuang, L. Schechter, Stability of experimental and survey measures of risk, time, and social preferences: A review and some new results, Journal of Development Economics 117 (2015) 151 - 170.

[3] S. Andersen, G. Harrison, M. I. Lau, E. Rutström, Eliciting risk and time preferences, Econometrica 76 (3) (2008) 583-618.

[4] G. W. Harrison, M. I. Lau, H. I. Yoo, Risk attitudes, sample selection and attrition in a longitudinal field experiment, The Review of Economics and Statistics (Forthcoming) 1-45.

[5] J. Lönnqvist, M. Verkasalo, G. Walkowitz, P. Wichardt, Measuring individual risk attitudes in the lab: Task or ask? an empirical comparison, Journal of Economic Behavior \& Organization 119 (2015) 254 - 266.

[6] S. Zeisberger, D. Vrecko, T. Langer, Measuring the time stability of prospect theory preferences, Theory and Decision 72 (3) (2012) 359-386. 
[7] F. Carlsson, O. Johansson-Stenman, P. K. Nam, Social preferences are stable over long periods of time, Journal of Public Economics 117 (2014) 104 114.

[8] A. C. M. de Oliveira, C. Eckel, R. T. A. Croson, The stability of social preferences in a low-income neighborhood, Southern Economic Journal 79 (1) (2012) 15-45.

[9] S. D. Levitt, J. A. List, What do laboratory experiments measuring social preferences reveal about the real world?, Journal of Economic Perspectives 21 (2) (2007) 153-174.

[10] S. Volk, C. Thöni, W. Ruigrok, Temporal stability and psychological foundations of cooperation preferences, Journal of Economic Behavior \& Organization 81 (2) (2012) $664-676$.

[11] S. Frederick, G. Loewenstein, T. O’Donoghue, Time discounting and time preference: A critical review, Journal of Economic Literature 40 (2) (2002) $351-401$.

[12] T. Tanaka, C. F. Camerer, Q. Nguyen, Risk and time preferences: Linking experimental and household survey data from Vietnam, American Economic Review 100 (1) (2010) 557-71.

[13] D. Cobb-Clark, S. Schurer, The stability of big-five personality traits, Economics Letters 115 (1) (2012) $11-15$.

[14] D. Cobb-Clark, S. Schurer, Two Economists' Musings on the Stability of Locus of Control, The Economic Journal 123 (570) (2013) F358-F400. 
[15] L. Borghans, J. Heckman, B. Golsteyn, H. Meijers, Gender Differences in Risk Aversion and Ambiguity Aversion, Journal of the European Economic Association 7 (2-3) (2009) 649-658.

[16] J. Heckman, R. Pinto, P. Savelyev, Understanding the mechanisms through which an influential early childhood program boosted adult outcomes, American Economic Review 103 (6) (2013) 2052-86.

[17] D. Arnott, S. Gao, Behavioral economics for decision support systems researchers, Decision Support Systems 122 (2019) 113063.

[18] J. Brandts, G. Charness, Hot vs. cold: Sequential responses and preference stability in experimental games, Experimental Economics 2 (3) (2000) 227 238.

[19] P. Crosetto, A. Filippin, A theoretical and experimental appraisal of four risk elicitation methods, Experimental Economics 19 (3) (2016) 613-641.

[20] C. Dave, C. C. Eckel, C. A. Johnson, C. Rojas, Eliciting risk preferences: When is simple better?, Journal of Risk and Uncertainty 41 (3) (2010) 219243.

[21] S. Ding, Z. Wang, D. Wu, D. L. Olson, Utilizing customer satisfaction in ranking prediction for personalized cloud service selection, Decision Support Systems 93 (2017) $1-10$.

[22] D. Geiger, M. Schader, Personalized task recommendation in crowdsourcing information systems: A current state of the art, Decision Support Systems 65 (2014) 3 - 16 . 
[23] S. Lim, B. Lee, Loyalty programs and dynamic consumer preference in online markets, Decision Support Systems 78 (2015) $104-112$.

[24] T. González-Artega, R. de Andrés Calle, M. Peral, Preference stability along time: the time cohesiveness measure, Progress in Artificial Intelligence 6 (3) (2017) 235-244.

[25] T. González-Arteaga, R. de Andrés Calle, New approach to measure preference stability, in: 2017 IEEE International Conference on Fuzzy Systems (FUZZ-IEEE), 2017, pp. 1-6.

[26] T. González-Arteaga, J. Cascón, R. de Andrés Calle, A proposal to measure human group behaviour stability, in: Information Processing and Management of Uncertainty in Knowledge-Based Systems. Applications 17th International Conference, IPMU 2018, 2018, pp. 99-110.

[27] L. Zadeh, Fuzzy sets, Information and Control 8 (1965) 338-375.

[28] J. Kelly, S. Qi, Balancedness of social choice correspondences, Mathematical Social Sciences 102 (2019) $59-67$.

[29] T. Rutar, For an integrative theory of social behaviour: Theorising with and beyond rational choice theory, Journal for the Theory of Social Behaviour 49 (3) (2019) 298-311.

[30] T. González-Arteaga, J. Alcantud, R. de Andrés Calle, A new consensus ranking approach for correlated ordinal information based on Mahalanobis distance, Information Sciences 372 (Supplement C) (2016) 546 - 564. 


\section{Appendix}

Proof of Lemma 1. It is trivial from Definition 2 and the definition of the permutation $\pi$ of the temporal decision profile, ${ }^{\pi} \mathcal{P}$.

Proof of Proposition 1. The result is a mere consequence of the following statement: Given a distance $d(\cdot, \cdot)$ based on a norm $\|\cdot\|$, for any $A \subseteq \mathbb{R}^{n}, \operatorname{diam}(A)=$ $\operatorname{diam}(\operatorname{Conv}(A))$ where $\operatorname{diam}(A)=\sup _{p, q \in A}\|p-q\|$. In this case, $A=\mathbf{F}$ and $\|\cdot\|=\|\cdot\|_{1}$.

Proof of Proposition 2. Let $\mathbf{c}, \mathbf{c}^{\prime} \in \mathbf{F}$ be two canonical codified temporal decisions such that $r=\left\|\mathbf{c}-\mathbf{c}^{\prime}\right\|_{1}$. There is no loss of generality in assuming that $\mathbf{c}$ is a non-increasing vector. This implies that $\mathbf{c}^{\prime}$ is a non-decreasing vector, because in other case, it could be permuted two non-ordered positions of $\mathbf{c}^{\prime}$, to produce $\overline{\mathbf{c}}$, and then a computation provides $\left\|\mathbf{c}-\mathbf{c}^{\prime}\right\|_{1} \leq \mathbf{c}-\overline{\mathbf{c}} \|_{1}$, that contradicts the choice of $\mathbf{c}$ and $\mathbf{c}^{\prime}$. By the characterization of the canonical codified preorder [30, Proposition 1], it is possible to infer: $c_{j} \in[k-j+1, k]$, and $c_{j}^{\prime} \in[j, k]$, for $j=1, \ldots, k$. Therefore:

$\left.\left\|\mathbf{c}-\mathbf{c}^{\prime}\right\|_{1}=\sum_{j=1}^{k}\left|c_{j}-c_{j}^{\prime}\right| \leq \sum_{j=1}^{k} \max \{k-j, k-(k-j+1)\} \leq \sum_{j=1}^{k} \max \{k-j, j-1)\right\}$.

The two values inside the maximum are complementary. The first is good for $j$ small, and the second is suitable for $j$ large. The crossover takes place for $j_{0}$ such that $k-j_{0} \leq j_{0}-1 \Rightarrow j_{0} \geq \frac{k+1}{2}$. Considering the parity of the number of 
alternatives:

Even $\left.k: \sum_{j=1}^{k} \max \{k-j, j-1)\right\}=\sum_{j=1}^{k / 2}(k-j)+\sum_{j=\frac{k}{2}+1}^{k}(j-1)=\frac{3}{4} k^{2}-\frac{k}{2}$,

Odd $\left.k: \sum_{j=1}^{k} \max \{k-j, j-1)\right\}=\sum_{j=1}^{(k-1) / 2}(k-j)+\sum_{j=\frac{k+1}{2}}^{k}(j-1)=\frac{3}{4} k^{2}-\frac{k}{2}-\frac{1}{4}$.

These results guarantee that $r$ is upper bounded by the expressions (1) and (2), respectively. The proof is completed by finding two feasible canonical codified temporal decisions of $\mathbf{F}$, whose distance is $r$. Motivated by the previous argument, we choose:

Even $k: \mathbf{c}=(\overbrace{k, k, \ldots, k}^{\frac{k}{2}-\text { times }}, \frac{k}{2}, \frac{k}{2}-1, \ldots, 1), \quad \mathbf{c}^{\prime}=(1,2, \ldots, \frac{k}{2}, \overbrace{k, k, \ldots, k}^{\frac{k}{2}-\text { times }})$

Odd $k: \mathbf{c}=(\overbrace{k, k, \ldots, k}^{\frac{(k-1)}{2}-\text { times }}, \frac{k+1}{2}, \frac{k-1}{2}, \ldots, 1), \quad \mathbf{c}^{\prime}=(1,2, \ldots, \frac{k-1}{2}, \overbrace{k, k, \ldots, k}^{\frac{(k-1)}{2}-\text { times }})$

This conclude the proof.

Proof of Lemma 2. By Definitions 2, 3 and assuming the permutation $\pi$ of the temporal decision profile ${ }^{\pi} \mathcal{P}$, the proof is immediate.

Proof of Proposition 3. Note that for $\lambda_{0}>0: \lim _{\lambda \rightarrow \lambda_{0}} \Theta(\mathcal{P}, \lambda)=\Theta\left(\mathcal{P}, \lambda_{0}\right)$. On the other hand, for $\lambda_{0}=0$, using L'Hôpital's rule it has been checked, $\lim _{\lambda \rightarrow 0}\left(w_{i, T}(\lambda)\right)=$ $\frac{1}{T}$, and therefore $\lim _{\lambda \rightarrow 0} \Theta(\mathcal{P}, \lambda)=\sum_{i=1}^{T} \frac{1}{T} \cdot \theta_{i}(\mathcal{P})=\Theta(\mathcal{P}, 0)$.

Proof of Compensativeness. Immediate from Definitions 2 and 3.

Proof of Convexity. Definition 3 is invoked to write

$\Theta(\mathcal{P}, \lambda)=\sum_{i=1}^{T} w_{i, T}(\lambda) \cdot \theta_{i}(\mathcal{P})=\sum_{j=1}^{T_{1}} w_{j, T}(\lambda) \cdot \theta_{j}\left(\mathcal{P}^{I_{1}}\right)+\sum_{j=1}^{T_{2}} w_{j+T_{1}, T}(\lambda) \cdot \theta_{j}\left(\mathcal{P}^{I_{2}}\right)$ 
On the one hand, examining the weight $\omega_{j, T}(\lambda)$, and since $T=T_{1}+T_{2}$

$$
\begin{aligned}
w_{j, T}(\lambda) & =A_{T}(\lambda) \cdot e^{-\lambda(T-i)}=A_{T}(\lambda) \cdot e^{-\lambda T_{2}} \cdot e^{-\lambda\left(T_{1}-i\right)} \\
& =\frac{A_{T}(\lambda)}{A_{T_{1}}(\lambda)} \cdot e^{-\lambda T_{2}} \cdot A_{T_{1}}(\lambda) \cdot e^{-\lambda\left(T_{1}-i\right)}=\frac{A_{T}(\lambda)}{A_{T_{1}}(\lambda)} \cdot e^{-\lambda T_{2}} \cdot \omega_{j, T_{1}}(\lambda) .
\end{aligned}
$$

From (3) it is possible to infer

$$
\frac{A_{T}(\lambda)}{A_{T_{1}}(\lambda)} \cdot e^{-\lambda T_{2}}=\left\{\begin{array}{ll}
\frac{1-e^{-\lambda T_{1}}}{1-e^{-\lambda T}} \cdot e^{-\lambda T_{2}}=\frac{e^{-\lambda\left(T-T_{1}\right)}-e^{-\lambda(T-0)}}{1-e^{-\lambda T}} & \text { if } \lambda \neq 0 \\
\frac{1 / T}{1 / T_{1}}=\frac{T_{1}}{T} & \text { if } \lambda=0
\end{array}\right\}=\alpha_{\lambda, T}\left(I_{1}\right) .
$$

On the other hand

$w_{j+T_{1}, T}(\lambda)=A_{T}(\lambda) \cdot e^{-\lambda\left(T-\left(j+T_{1}\right)\right)}=\frac{A_{T}(\lambda)}{A_{T_{2}}(\lambda)} \cdot A_{T_{2}}(\lambda) \cdot e^{-\lambda\left(T_{2}-j\right)}=\frac{A_{T}(\lambda)}{A_{T_{2}}(\lambda)} \cdot \omega_{j, T_{2}}(\lambda)$

and again from (3) it is obtained

$$
\frac{A_{T}(\lambda)}{A_{T_{2}}(\lambda)}=\left\{\begin{array}{ll}
\frac{1-e^{-\lambda T_{2}}}{1-e^{-\lambda T}}=\frac{e^{-\lambda\left(T-\left(T_{1}+T_{2}\right)\right)}-e^{-\lambda\left(T-T_{1}\right)}}{1-e^{-\lambda T}} & \text { if } \lambda \neq 0 \\
\frac{1 / T}{1 / T_{2}}=\frac{T_{2}}{T} & \text { if } \lambda=0
\end{array}\right\}=\alpha_{\lambda, T}\left(I_{2}\right)
$$

Collecting the previous results we conclude the proof.

Proof of No Timelessness. The proof is a straightforward from Definitions 2 and 3.

Proof of Convergence full stability. The extended decision temporal profile $\overline{\mathcal{P}}^{q}$ can be decomposed into two temporal decision subprofiles such that:

$$
\overline{\mathcal{P}}^{q}=\left(\mathcal{P}^{I_{1}}, \mathcal{P}^{I_{2}}\right)=(\overbrace{\mathcal{R}_{0}, \ldots, \mathcal{R}_{T}, \underbrace{\mathcal{R}_{T+1}^{I_{1}}}_{\mathcal{P}^{I_{2}}}, \mathcal{R}_{T+2}, \ldots, \mathcal{R}_{T+q}} .) \in \bigcup_{T \geq 1} \mathbf{W}(\mathbf{X})^{T+1+q}
$$

then, using the convexity property with $I_{1}=\left\{t_{0}, \ldots, t_{T}, t_{T+1}\right\}$, and $I_{2}=\left\{t_{T+1}, \ldots, t_{T+q}\right\}$ :

$$
\begin{aligned}
& \Theta\left(\overline{\mathcal{P}}^{q}, \lambda\right)=\alpha_{\lambda, T}\left(I_{1}\right) \cdot \Theta\left(\mathcal{P}^{I_{1}}, \lambda\right)+\alpha_{\lambda, T}\left(I_{2}\right) \cdot \Theta\left(\mathcal{P}^{I_{2}}, \lambda\right) \\
& \alpha_{\lambda, T}\left(I_{1}\right)=\frac{e^{-\lambda(q-1)}-e^{-\lambda(T+q)}}{1-e^{-\lambda(T+q)}}, \quad \alpha_{\lambda, T}\left(I_{2}\right)=\frac{1-e^{-\lambda(q-1)}}{1-e^{-\lambda(T+q)}}
\end{aligned}
$$


Taking the limit: $\lim _{q \rightarrow \infty} \alpha_{\lambda, T}\left(I_{1}\right)=0, \lim _{q \rightarrow \infty} \alpha_{\lambda, T}\left(I_{2}\right)=1$. Since $\Theta\left(\mathcal{P}^{I_{2}}, \lambda\right)=$ 1 because $\mathcal{R}_{T+1}=\ldots=\mathcal{R}_{T+q}$ and $\Theta\left(\mathcal{P}^{I_{1}}, \lambda\right)$ does not depended on $q$, it is possible to infer the desired result.

Proof of Absolute monotonicity. Let $\overline{\mathcal{P}}=\left(\mathcal{R}_{0}, \ldots, \mathcal{R}_{T}, \mathcal{R}_{T+1}\right) \in \bigcup_{T \geq 1} \mathbf{W}(\mathbf{X})^{T+2}$ an extended decision temporal profile. Consider the following overlapped partition on the extended temporal set $\overline{\mathbf{T}}=\left\{t_{0}, \ldots, t_{T}, t_{T+1}\right\}, I_{1}=\left\{t_{0}, \ldots, t_{T}\right\}$ and $I_{2}=\left\{t_{T}, t_{T+1}\right\}$ that generates two consecutive temporal decision subprofiles $\overline{\mathcal{P}}^{I_{1}}=\mathcal{P}$ and $\overline{\mathcal{P}}^{I_{2}}, \overline{\mathcal{P}}^{I_{1} \cup I_{2}}=\overline{\mathcal{P}}$. By convexity property: $\Theta(\overline{\mathcal{P}}, \lambda)=\alpha_{\lambda, T}\left(I_{1}\right)$. $\Theta(\mathcal{P}, \lambda)+\alpha_{\lambda, T}\left(I_{2}\right) \cdot \theta_{T+1}\left(\overline{\mathcal{P}}^{I_{2}}\right)$. Taking into account that $\mathcal{R}_{T}=\mathcal{R}_{T+1}$ and Definition 2: $\left\|\mathbf{c}_{\mathcal{R}_{T}}-\mathbf{c}_{\mathcal{R}_{T+1}}\right\|_{1}=0$ and then $\theta_{T+1}\left(\overline{\mathcal{P}}^{I_{2}}\right)=1$, consequently, $\Theta(\overline{\mathcal{P}}, \lambda) \in(\Theta(\mathcal{P}, \lambda), 1)$.

Proof of Relative monotonicity. This proof is similar to the previous one and will be omitted.

Proof of Comparative monotonicity.. Let $\overline{\mathcal{P}}=\left(\mathcal{R}_{0}, \ldots, \mathcal{R}_{T}, \mathcal{R}_{T+1}\right), \overline{\mathcal{P}}^{\prime}=\left(\mathcal{R}_{0}, \ldots, \mathcal{R}_{T}, \mathcal{R}_{T+1}^{\prime}\right) \in$ $\bigcup_{T \geq 1} \mathbf{W}(\mathbf{X})^{T+2}$ be two extended temporal decision profiles. Consider the overlapped partition on the set $\overline{\mathbf{T}}=\left\{t_{0}, \ldots, t_{T}, t_{T+1}\right\}, I_{1}=\left\{t_{0}, \ldots, t_{T}\right\}$ and $I_{2}=$ $\left\{t_{T}, t_{T+1}\right\}$ that generates two consecutive temporal decision subprofiles for each extended temporal profile:

$$
\begin{aligned}
& \overline{\mathcal{P}}^{I_{1}}=\mathcal{P} \quad \text { and } \quad \overline{\mathcal{P}}^{I_{2}}, \quad \overline{\mathcal{P}}^{\left(I_{1} \cup I_{2}\right)}=\overline{\mathcal{P}} \text { for } \quad \overline{\mathcal{P}}
\end{aligned}
$$

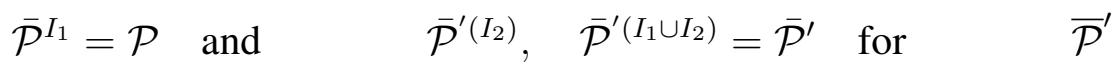

By convexity property:

$$
\begin{gathered}
\Theta(\overline{\mathcal{P}}, \lambda)=\alpha_{\lambda, T}\left(I_{1}\right) \cdot \Theta(\mathcal{P}, \lambda)+\alpha_{\lambda, T}\left(I_{2}\right) \cdot \theta_{T+1}\left(\overline{\mathcal{P}}^{I_{2}}\right) \\
\Theta\left(\overline{\mathcal{P}}^{\prime}, \lambda\right)=\alpha_{\lambda, T}\left(I_{1}\right) \cdot \Theta(\mathcal{P}, \lambda)+\alpha_{\lambda, T}\left(I_{2}\right) \cdot \theta_{T+1}\left(\overline{\mathcal{P}}^{\prime}\left(I_{2}\right)\right.
\end{gathered}
$$


Considering $\left\|\mathbf{c}_{\mathcal{R}_{T}}-\mathbf{c}_{\mathcal{R}_{T+1}^{\prime}}\right\|_{1}>\left\|\mathbf{c}_{\mathcal{R}_{T}}-\mathbf{c}_{\mathcal{R}_{T+1}}\right\|_{1}$ and Definition 2, it is possible to conclude: $\theta_{T+1}\left(\overline{\mathcal{P}}^{I_{2}}\right)>\theta_{T+1}\left(\overline{\mathcal{P}}^{\prime}\left(I_{2}\right)\right)$, consequently, $\Theta(\overline{\mathcal{P}}, \lambda)>\Theta\left(\overline{\mathcal{P}}^{\prime}, \lambda\right)$.

Proof of Decision monotonicity.. Let $\mathbf{c}_{\mathcal{R}_{i}}=\left(c_{1}^{\mathcal{R}_{i}}, \ldots, c_{k}^{\mathcal{R}_{i}}\right)$ and $\mathbf{c}_{\widetilde{\mathcal{R}}_{i}}=\left(c_{1}^{\widetilde{\mathcal{R}}_{i}}, \ldots, c_{k}^{\widetilde{\mathcal{R}}_{i}}, \ldots, c_{k+q}^{\widetilde{\mathcal{R}}_{i}}\right)$ be the canonical codifications of $\mathcal{R}_{i}$ and $\widetilde{\mathcal{R}}_{i}$, respectively, for $i=0, \ldots T$. Note that the value of each coefficient of $\mathbf{c}_{\mathcal{R}_{i}}\left(\right.$ or $\mathbf{c}_{\widetilde{\mathcal{R}}_{i}}$ ), is given by ${ }^{14}$ :

$$
c_{j}^{\mathcal{R}_{i}}=\left|\left\{r: x_{j} \mathcal{R}_{i} x_{r}\right\}\right|,
$$

therefore, conditions (a) and (b) imply:

$$
\begin{aligned}
& 11 c_{j}^{\tilde{\mathcal{R}}_{i}}=c_{j}^{\tilde{\mathcal{R}}_{i}} \text { for all } j \in\{k+1, \ldots, k+q\} \text { and } i \in\{0, \ldots, T\} . \\
& 2 c_{j}^{\widetilde{\mathcal{R}}_{i}}= \begin{cases}c_{j}^{\mathcal{R}_{i}} & \text { if the new alternatives are better graded, } \\
c_{j}^{\mathcal{R}_{i}}+q & \text { if the new alternatives are worse graded. }\end{cases}
\end{aligned}
$$

In any case, it is obtained: $\left\|\mathbf{c}_{\tilde{\mathcal{R}}_{i-1}}-\mathbf{c}_{\widetilde{\mathcal{R}}_{i}}\right\|_{1}=\left\|\mathbf{c}_{\mathcal{R}_{i-1}}-\mathbf{c}_{\mathcal{R}_{i}}\right\|_{1}$, for all $i=\{1, \ldots, T\}$. Using this result and the fact that $r<\widehat{r}$, it is got:

$$
\theta_{i}(\mathcal{P})=1-\frac{\left\|\mathbf{c}_{\widetilde{\mathcal{R}}_{i-1}}-\mathbf{c}_{\widetilde{\mathcal{R}}_{i}}\right\|_{1}}{r}<1-\frac{\left\|\mathbf{c}_{\mathcal{R}_{i-1}}-\mathbf{c}_{\mathcal{R}_{i}}\right\|_{1}}{\widetilde{r}}=\theta_{i}(\widetilde{\mathcal{P}})
$$

for all $i \in\{1, \ldots, T\}$ and consequently, $\Theta(\mathcal{P}, \lambda)<\Theta(\widetilde{\mathcal{P}}, \lambda)$.

Proof of Proposition 4. The proof is by induction on $s$. The case $s=2$ is the convexity property that it was early established ${ }^{15}$. Assuming the case $s-1$ and it is shown that (6) holds for $s$. Since $\left\{I_{1}, \ldots, I_{s}\right\}$ is an overlapped partition, it is

\footnotetext{
${ }^{14}$ see González-Arteaga, Alcantud and de Andrés Calle [30].

${ }^{15}$ If $s=2$, i.e., the convexity property, $T_{k}^{+}=T_{1}$ and $T_{k}^{-}=0$ for $k=1$; and $T_{k}^{+}=T_{1}+T_{2}=T$ and $T_{k}^{-}=T_{1}$ for $k=2$.
} 
obtained:

$$
\left|I_{k}\right|=T_{k}+1, \quad \text { for } k=1, \ldots, s, \quad T_{1}+T_{2}+\ldots+T_{s}=T
$$

Firstly, an auxiliary overlapped partition is defined $\left\{J_{1}, J_{2}\right\}$ given by: $J_{1}=I_{1} \cup$ $\ldots \cup I_{s-1}$ and $J_{2}=I_{s}$. The cardinality of these new sets are $\left|J_{1}\right|=1+\sum_{k=1}^{s-1} T_{k}$, or equivalently $\left|J_{1}\right|=1+\left(T-T_{s}\right)$ and $\left|J_{2}\right|=I_{s}$. Secondly, the convexity property (4) is invoked and then, by the induction hypothesis it is possible to write:

$$
\begin{aligned}
\Theta(\mathcal{P}, \lambda) & =\alpha_{\lambda, T}\left(J_{1}\right) \cdot \Theta\left(\mathcal{P}^{J_{1}}, \lambda\right)+\alpha_{\lambda, T}\left(J_{2}\right) \cdot \Theta\left(\mathcal{P}^{J_{2}}, \lambda\right) \\
& =\alpha_{\lambda, T}\left(J_{1}\right)\left(\sum_{k=1}^{s-1} \alpha_{\lambda,\left(T-T_{s}\right)}\left(I_{k}\right) \cdot \Theta\left(\mathcal{P}^{I_{k}}, \lambda\right)\right)+\alpha_{\lambda, T}\left(I_{s}\right) \cdot \Theta\left(\mathcal{P}^{I_{s}}, \lambda\right)
\end{aligned}
$$

Now the product of $\alpha$-weights is studied, i.e., $\alpha_{\lambda, T}\left(J_{1}\right) \cdot \alpha_{\lambda,\left(T-T_{s}\right)}\left(I_{k}\right)$. From (7), assuming initially $\lambda>0$ and remaining that the upper time index, $T_{J_{1}}^{+}$, and lower time index, $T_{J_{1}}^{-}$, of the set $J_{1}$ are $\sum_{j=1}^{s-1} T_{j}$ and 0 , respectively, some simply computations provide $\alpha_{\lambda, T}\left(J_{1}\right) \cdot \alpha_{\lambda,\left(T-T_{s}\right)}\left(I_{k}\right)=\alpha_{\lambda, T}\left(I_{k}\right)$. The case $\lambda=0$ is simpler and it can be omitted. Therefore, the statement (6) is consequence of the two previous results. Finally, to get $\sum_{k=1}^{s} \alpha_{\lambda, T}\left(I_{k}\right)=1$, note that the numerators of $\alpha_{\lambda, T}(\cdot)$ are a telescoping series ${ }^{16}$.

\footnotetext{
${ }^{16} \mathrm{~A}$ series whose partial sums have a fixed number of terms after their cancellation (see T. M. Apostol, Calculus, Volume 1, Blaisdell Publishing Company).
} 